\section{Thermal Analysis of Space Debris for Infrared Based Active Debris Removal}

Proc IMechE Part G: J Aerospace Engineering $\mathrm{XX}(\mathrm{X}): 1-13$ (C)The Author(s) 2017 Reprints and permission: sagepub.co.uk/journalsPermissions.nav DOI: 10.1177/ToBeAssigned www.sagepub.com/

@SAGE

\author{
Özgün Yılmaz ${ }^{1}$, Nabil Aouf ${ }^{1}$, Elena Checa ${ }^{2}$, Laurent Majewski ${ }^{3}$ and Manuel \\ Sanchez-Gestido ${ }^{2}$
}

\begin{abstract}
In space, visual based relative navigation systems suffer from dynamic illumination conditions of the target (Eclipse conditions, solar glare...etc.) where most of these issues are addressed by advanced mission planning techniques. However, such planning would not be always feasible or even if it is, it would not be straightforward for Active Debris Removal (ADR) missions. On the other hand, using an infrared based system would overcome this problem, if a guideline to predict infrared signature of space debris based on the target thermal profile could be provided for algorithm design and testing.

Spacecraft thermal design is unique to every platform. This means every ADR target will have a different infrared signature which changes over time not just only due to orbital dynamics but also due to its thermal surface coatings. In order to provide a space debris infrared signature guideline for most of the possible ADR targets, we introduce an innovative grouping system for thermal surface coatings based on their behaviour in Space environment. Through the use of this grouping system, we propose a space debris infrared signature estimation method which was extensively verified by our simulations and experiments. During our verifications, we have also found out very important problem so called "Signature Ambiguity" that is unique to Infrared Based Active Debris Removal (IR-ADR) systems which we have also discussed in our work.
\end{abstract}

\title{
Keywords
}

Space Debris, Infrared Imaging, Infrared Signature, Thermal Analysis, Active Debris Removal, Relative Navigation

\section{Introduction}

Uncontrolled growth of orbital debris has been shown to be a significant threat to operational satellites and human activities in space ${ }^{12}$. Some studies ${ }^{34}$ state that a significant amount of these orbital threats is around the Low Earth Orbit (LEO), making it the most vulnerable region in terms of orbital exposure to collision threats. It has been stated that simply monitoring the space environment from ground ${ }^{5}$ and space based observations ${ }^{6}$ is no longer sufficient to prevent losses of space missions as a result of orbital collisions ${ }^{7}$. Therefore, there is a clear requirement to reduce or at least limit the amount of space debris in orbit.

Active Debris Removal (ADR) is a mission concept where a chaser spacecraft captures a space debris, such as a non-operational spacecraft, which is potentially a significant threat for other operational man-made orbiting bodies $^{7}$. Even though there have been some studies on ADR technologies ${ }^{89}$, there are still some more technical issues to overcome ${ }^{10}$ before an ADR can actually be demonstrated. Among them, one of the most important technical challenges is the relative navigation towards an uncooperative target ${ }^{11}$ (non-operational spacecraft without fiducial markers and without attitude and orbit control, therefore possibly tumbling e.g. ENVISAT ${ }^{12}$ ). After then, the chaser capture mechanism would be able to grab the target and apply the relevant disposal activities.

The relative navigation with LIDAR, RADAR and visual based systems has been already well studied and supporting the current Rendezvous and Docking (RvD) missions ${ }^{13}$.

\footnotetext{
${ }^{1}$ Department of Electronic Warfare, Cranfield University, United Kingdom ${ }^{2}$ ESTEC, European Space Agency, The Netherlands

${ }^{3}$ Architects and Optronic Systems, SODERN, France

\section{Corresponding author:}

Özgün Yılmaz, MH214 Defence Academy of United Kingdom, Shrivenham, SN6 8LA, UK.

Email: o.yilmaz@cranfield.ac.uk
} 
The RADAR systems provide information about the target distance and direction (angle) mainly during the long and medium ranges by evaluating the echo of emitted RF signals ${ }^{14}$ with possible additional information of target structure $^{15}$. Although they can also operate in a very short range, achieving the required performance is relatively more difficult therefore not preferred in close proximity operations (final part of Short-Range Rendezvous Phase) ${ }^{13}$. LIDAR is another active system that has been used for retrieving the range and the target structure information however its performance may decrease at very close range due to target structure observed like a planar surface. In the case of $\mathrm{RvD}$, the target is generally cooperative and has some retroreflectors to increase the visibility by reflecting most of the signal to the emitter where these aids enable quicker processing and identification ${ }^{13} 16$. Some recent experimental studies showed that the operation range of LIDAR systems for Space Rendezvous against non-cooperative targets can go up to $260 \mathrm{~m}^{17}$. Visual systems are the passive sensor alternative of the LIDAR and RADAR approaches and are heavily used in close proximity operations. Their measurement accuracy can be $2 \mathrm{~cm}$ or better in relative range and 0.05 degree or better in relative attitude at the range of 2 meters with a cooperative target ${ }^{18}$. However, visual systems also have some drawbacks in terms of target illumination conditions, such as being non-operational under eclipse conditions or being affected by solar glare in a space environment. The flight demonstration undertaken by ${ }^{19}$ underlines the need for a sufficient level of illumination. In current RvD demonstrations, this situation is avoided in mission design by using different approaches to client spacecraft, planning the manoeuvres so that certain Suntarget-chaser geometries are ensured ${ }^{1320}$.

In the unknown environment of ADR, Sun avoidance in the sensor field of view and target illumination conditions cannot be imposed and most likely the conditions would vary dramatically ${ }^{21}$. On the other hand, infrared based systems could provide the information required when all these sensors fail or are not applicable since the working principle of infrared detectors is based on the fact that all objects with a temperature greater than absolute zero radiate in addition to reflecting the infrared radiation of other sources. Even though infrared based relative navigation is fairly a new concept, there have been few studies on their possible usage as a complementary sensor in uncooperative rendezvous like in Ref. ${ }^{22}$ Ref. $^{8}$ Ref. $^{23}$ and some in-situ measurements have been collected in few experiments Ref. ${ }^{24}$ Ref. ${ }^{25}$ Ref. ${ }^{20}$.
Unfortunately infrared environment present in ADR is still not well known and yet there is no fully verified infrared simulation environment available which may lead to over simplified assumptions and therefore mislead the further studies. For instance, Ref. ${ }^{26}$ proposed an uncooperative target pose estimation method using infrared camera based on the assumption of static, very bright targets which seem to be too optimistic for the thermally dynamic environment of ADR. Therefore, the performance of their proposed method to a real application cannot be evaluated.

In infrared imaging, the target scene temperature range plays an important role in choosing the correct detector waveband which maximises the amount of received signals and provides better SNR for detection algorithms. As ADR infrared detector, there are five possible options: Near-Infrared (NIR), Short-Wave Infrared (SWIR), MidWave Infrared (MWIR), Long-Wave Infrared (LWIR), and Far-Infrared (FIR). Therefore, the target (Space Debris) temperature profile is important to know.

In this context, the infrared characteristics of Space Debris have to be well understood in order to develop IRADR algorithms which would not only work in simulation environment but also in real scenarios. The infrared signatures are function of the object temperatures and reflected radiation coming from other infrared sources. Therefore one should know the temperature profile of space debris to estimate the amount of infrared radiation. For the thermal analysis of Space Debris, Ref. ${ }^{27}$ had already performed some studies based on Finite Element Analysis. However this analysis has been found cumbersome and computationally heavy to be used or applied to infrared signature analysis of ADR targets for relative navigation in terms of practicality. In a further study, Ref. ${ }^{28}$ investigated another approach for debris characterisation in infrared spectrum where specific spacecraft surface coating materials were analysed. Even though both provided some insights to ADR infrared environment, they were still not generic enough to be used in a verification and validation process of any infrared based navigation solution. The performed works evaluated the problem from spacecraft thermal design point of view rather than infrared imaging which made it difficult and not applicable for the ultimate goal of our study (Thermal analysis to estimate space debris infrared signature).

In our study, considering the debris population and the benefits of infrared systems, LEO had been selected as the target orbit. We then sampled LEO with three different eclipse conditions that matters for infrared Imaging and named as Orbit Type-I, Orbit Type-II and Orbit Type-III. 
By using a thermal approximation method we estimated their thermal profile in which we propose the best infrared band applicable for most of the ADR scenarios. Based on our thermal analysis, we suggested possible ambiguities in infrared signatures and problems relevant to IR-ADR. Finally, we simulated our analysis for a sample case then described the expected thermal scene structure and the challenges. The study outcome provides a practical procedure to simulate a representative thermal environment for different ADR targets so that a robust infrared based navigation solution can be developed for realistic conditions and be tested considering all relevant challenges.

\section{Selection of The Orbit Types}

In the scope of this study, the term "space debris" has been used to describe a spacecraft without any orbital and attitude control system that is orbiting around the Earth. In addition, they have been assumed to be tumbling as they do not have attitude control. Therefore they can be considered as orbiting bodies without any internal torque or force to change their orbit and attitude instantaneously. The objects orbital period $(T)$ depends on the semi-major axis $(a)$ of the orbit and the standard gravitational parameter $(\mu)$ which is a function of the central body mass ${ }^{29}$.

$$
T=2 \pi \sqrt{\frac{a^{3}}{\mu}}
$$

The orbiting bodies experience eclipse if their orbital plane crosses the umbra cone (which is the case for most of the man-made orbiting objects in LEO). The duration and the frequency of eclipse depend on the orbital period and the geometry of the orbital plane respect to Sun. Therefore, the altitude -for circular orbits- of the orbiting body defines the frequency of the eclipse and indirectly the duration of eclipse. This means that for an object in low altitude, every 100 minutes there could be up to $\sim 30$ minutes of eclipse while at higher orbits, the eclipse duration can go up to $\sim 1$ hour where the orbital period is $\sim 24$ hours. In practice, the interest of placing a spacecraft in higher orbits mainly focuses on low inclinations where these long durations of eclipses happen at only specific times of the year.

In other words by launching ADR Mission for targets at very high orbits at specific times of the year -which is a fairly large window-, the eclipse can be avoided. Therefore, the advantage of infrared imagers would not be so significant since the solar glare is not as frequent phenomena as eclipse most of the time. On the other hand considering the durations of RvD missions ${ }^{13}$, eclipse cannot be avoided for Low Earth
Orbiting (LEO) objects by such means and the advantage of infrared based system would be significant comparing to using a visual based solution.

With this conclusion, this study only focuses on infrared signatures of LEO Space Debris for three different representative cases named as Orbit Type-I, Orbit TypeII and Orbit Type-III. The Orbit Type-I represents the case of an orbit without eclipse when the orbital plane is perpendicular to Earth-Sun pointing vector whereas Orbit Type-II represents the orbit of 100 minutes with around 30 minutes eclipse. It is important to highlight the fact that these orbital regions are particular cases called Sun Synchronous Orbits (SSO) and heavily used in Earth observation missions ${ }^{30}$. Orbit Type-III had been just chosen to understand the effect of eclipse duration in thermal analysis and therefore constructed in such a way to have half of the Orbit Type-II eclipse duration. All of these orbit types are considered to be at same altitude and therefore with same orbital period but with different duration of eclipse.

For the generality of the study, we would also like to mention that the performed analysis can be extended with few modifications to higher orbits. The effect of orbital dynamics on thermal variations would not much differ from lower orbits for worst case scenario (only the frequency of eclipse would decrease). However, the incoming thermal flux parameters have to be recalculated according to their chosen altitude.

\section{Thermal Approximation of Space Environment for Thermal Analysis}

The temperature of an object is a function of heat flux difference between net income and the total radiated power. Since the space debris does not have neither power dissipation nor active cooling, incoming heat fluxes would only be from the Sun and Earth, where the thermal equilibrium relies on the passive thermal design of spacecraft. For a tumbling space debris with a rate other than orbit rate, the direction of these heat influxes would change over time and therefore would not heat only one side or face.

As a common practice, spacecraft thermal designs have been performed in such a manner that they satisfy the homogeneous radiant heat transfer within the body ${ }^{31}$. Therefore, space debris could be thought as isothermal object where there is no high temperature gradients within the body. According to Finite Element Analysis of ${ }^{27}$, Radiative Thermal Equilibrium (RTE) temperatures of an isothermal tumbling object is independent from its tumbling rate. If the tumbling rate is faster than $0.01 \mathrm{rpm}$ (where the 
orbital rate is $\sim 0.06^{\circ} / s$ ), there would not be a significant temperature difference between the faces of the satellite (if it is considered as a cube) since the heat influx would be similar. This would enable us to simplify the complex geometry of the tumbling space debris to an isothermal unit sphere and to make the assumption of homogeneous incidence of environmental heat fluxes on debris surface.

With all these assumptions, the total amount of heat flux under Sun and eclipse conditions can be simplified as the following

$$
\begin{gathered}
Q_{S T \text { tol }}=Q_{S}+Q_{A}+Q_{I R} \\
Q_{\text {ETotal }}=Q_{I R}
\end{gathered}
$$

where $Q_{S}$ is solar flux, $Q_{A}$ is Earth albedo flux, $Q_{I R}$ is flux from Earth infrared (IR) radiation.

In steady state conditions, if the surface would be covered with only one thermal coating the temperature of isothermal space debris would be ${ }^{32}$

$$
T_{E}=\sqrt[4]{\frac{Q_{\text {Total }}}{\varepsilon A \sigma}}
$$

where $\varepsilon$ is the emissivity of the coating material, $A$ is the cross sectional area for radiation exchange, $\sigma$ is StefanBoltzmann constant, $T_{E}$ is the equilibrium temperature, $Q_{\text {Total }}$ is the total absorbed energy by the body in steady state conditions.

As outlined previously, lower altitude orbits cross the umbra cone more often than higher altitudes due to the orbital dynamics. Therefore, depending on the thermal inertia of the surface coating material the object surface might not reach to equilibrium temperature. The thermal inertia is a measure of how fast the material reacts the changes in thermal environment.

Instead of performing computationally heavy Finite Element Analysis (FEA) for a limited number of orbital cases shown in Ref. ${ }^{27}$, the analysis Simple Thermal Analytical Models (STAM) presented in Ref. ${ }^{32}$ has been used to evaluate the thermal variations throughout the orbit of different types that was described earlier.

According to Ref. ${ }^{32}$, the actual surface temperature of each coating could be approximated by assuming pure radiation and cooling for isothermal body as the following

$$
C \frac{d T}{d t}=\varepsilon A \sigma\left(T_{E}^{4}-T^{4}\right)
$$

where $C$ is thermal capacitance which is the merit of thermal inertia and can be solved as:

$$
\frac{T}{T_{E}}=1+\left(\frac{T_{O}}{T_{E}}-1\right)^{\frac{-t}{\tau}}
$$

where $T_{O}$ is the starting temperature and

$$
\tau=\frac{C}{4 \varepsilon A \sigma T_{E}^{3}}
$$

This approximation is verified with ESATAN-TMS FEA ${ }^{33}$ tool by using the same parameters of considered materials. ESATAN-TMS FEA is a finite element thermal analysis software that is used in spacecraft thermal design. The results (Figure 1) show that although the temporal variations of external heat flux have been heavily smoothed in our analysis, the trend of ESATAN-TMS FEA has still been tracked reasonably well.

As it can be seen from Ref. ${ }^{32}$ approximation, the surface temperature relies on the thermal inertia, emissivity and indirectly absorptivity (through absorbed influx by the surface) of the coating material.

In space applications, the most common external surface covers are the radiator coatings, insulation blankets (such as MLI) and paints ${ }^{31}$. The insulation coatings for external surfaces usually have high emittance with varying absorptivity values depending on the application need ${ }^{31}$. However, the coatings with high absorptance-to-emittance ratio $(\alpha / \varepsilon)$ are not preferred in exterior surfaces of manmade space objects as they create hot spots on the structure $^{31}$.

Our study aims to characterize the behaviour of typical external space debris surface coatings for infrared imaging. In this context, space coatings were grouped as in Table- 1 for the purpose of the study. The term thermal inertia shown in Table 1 has been linked to the time response $(\tau)$ of materials to the variations in heat flux. High thermal inertia means the material is resistant to thermal fluctuations whereas low thermal inertia materials respond fast for thermal variations in the environment. For "very low inertia", "low inertia" and "high inertia" materials, $\tau$ values are considered in the order of 0-100 seconds, couple of hundred to a thousand seconds and couple of thousand seconds respectively.

Table 1. Classification of thermal properties for spacecraft typical external surface materials

\begin{tabular}{lll}
\hline Thermal Inertia & $\alpha / \varepsilon \sim 1$ & $\alpha / \varepsilon \ll 1$ \\
\hline High & SAR, MMOD & Radiators \\
Low & Solar Panels & White Paint \\
Very Low & MLI & Beta Cloth \\
\hline
\end{tabular}

Assuming that each isothermal sphere with unit surface area is covered by only one kind of thermal coatings given in Table 1, the thermal analyses were performed for the three 


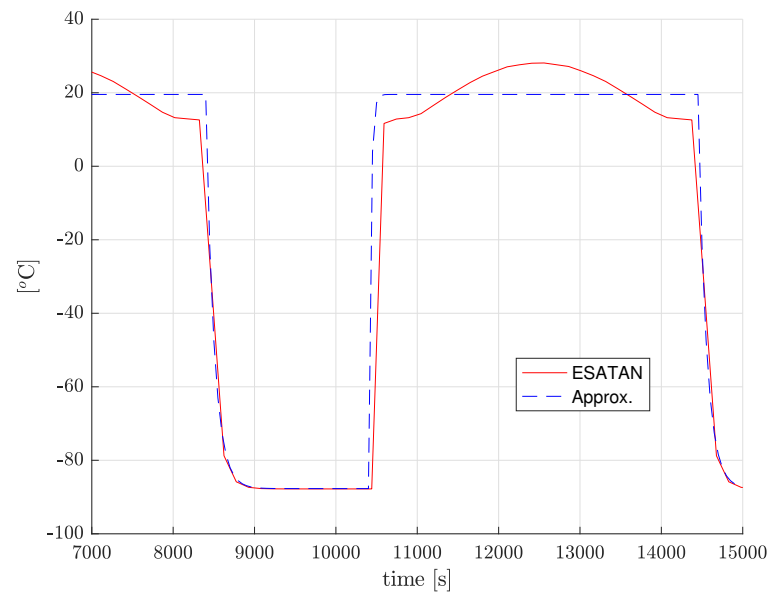

(a)

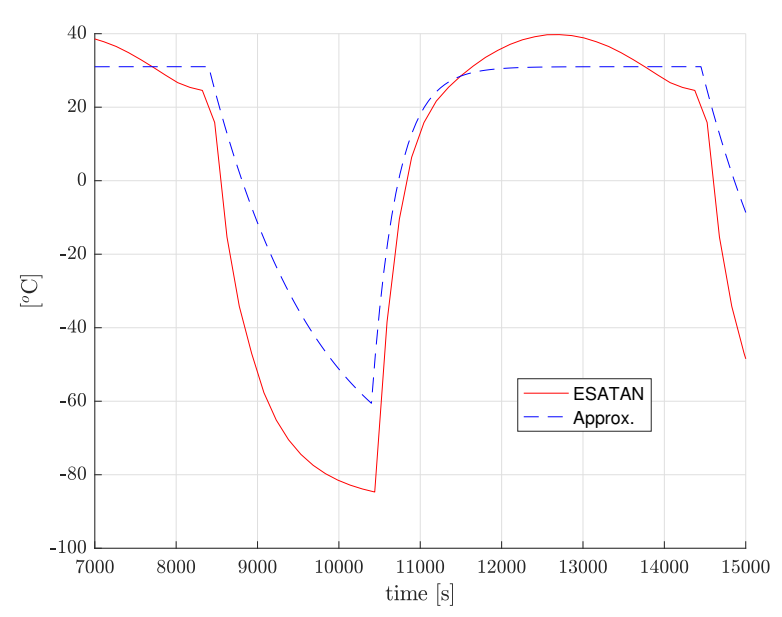

(b)

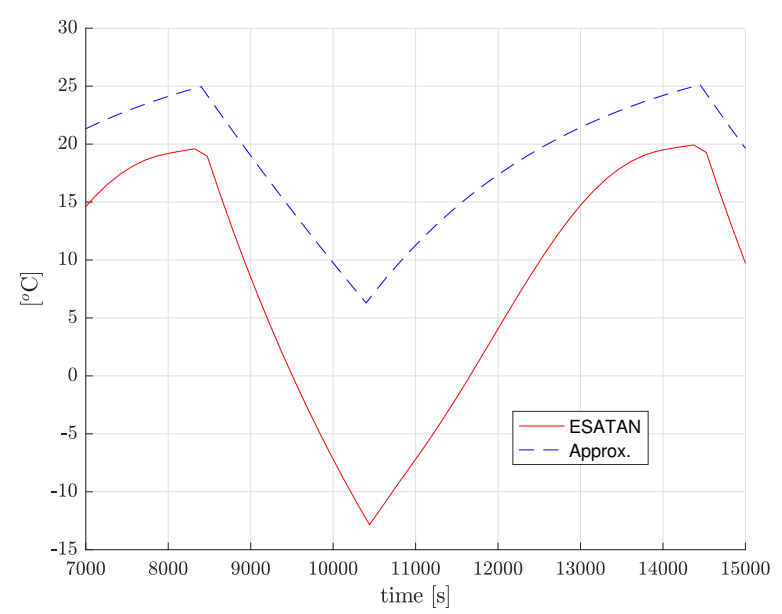

(c)

Fig. 1 Comparing "Simple Thermal Analytical Models"32 approximation to ESATAN-TMS FEA for materials with (a) Very Low Inertia, (b) Low Inertia and (c) High Thermal Inertia.

types of orbits (Orbit Type-I, Orbit Type-II and Orbit TypeIII).

\section{Thermal Analysis of Space Debris for Different Orbits}

Depending on the orbital parameters, the space debris/objects can experience different thermal profiles along their orbit. In the case of space debris where there is no internal power dissipation, this thermal profile solely depends on the external heat fluxes of which solar radiation is the most significant. In order to quantify such effect for infrared imaging system's perspective, the study evaluated three different orbital types whose altitudes were same -therefore the orbital periods- but the orbital geometries respect to Sun and Earth differed.

In order to do so, the analysis assumed that all representative orbits were circular with the apogee of $800 \mathrm{~km}$. Corresponding Solar-IR and Earth-IR flux densities were approximated as $350 \mathrm{~W} / \mathrm{m}^{2}$ and $65 \mathrm{~W} / \mathrm{m}^{2}$ respectively. The given values were derived from the mean of ESATAN-TMS computational results for given orbital parameters. Although the computational results showed yearly variations in solar influx, they were found to be negligible for the ultimate goal of the study. However, the albedo flux which was required for computing the total absorbed heat flux, would differ in each case as it depends on the Sun-Earth-Target geometry which is indirectly linked to the orbital geometry. Therefore, the albedo fluxes which are present on the illuminated side of Earth, were computed separately for each representative orbit.

\subsection{Orbit Type-I}

In some particular orbits such as Dawn-Dusk SSO, the orbiting bodies do not cross the umbra cone and therefore always expose to solar radiation. Therefore, the space debris tend to be relatively warmer comparing to other geometries and shows almost a stable thermal profile. The only thermal variations in orbit were expected to be caused by Earth's albedo flux as it's a function of Sun-Earth-Target angle and applicable for the illuminated part of the Earth. Considering the geometry, the albedo flux density of the represented orbit was approximated as $20 \mathrm{~W} / \mathrm{m}^{2}$ from the mean values of ESATAN-TMS computations for one orbit.

Figure 2 shows the computed thermal profile of the Orbit Type-I. Since there was no eclipse in this orbit, temperature profile was observed to be in steady state conditions due to the constant influx coming from Earth and the Sun. However, the temperature profiles of different coating materials in Figure 2 were observed to be divided into two groups, based on their $\alpha / \varepsilon$ ratios. This suggested that in such orbit, the temperature profile of an object does not depend on the 
thermal inertia of the material but the ratio of $\alpha$ (solar absorptivity) and $\varepsilon$ (emissivity). The materials with $\alpha / \varepsilon \ll 1$ such as "radiators" tend to be a lot cooler than materials with $\alpha / \varepsilon \sim 1$ such as "solar panels". Such results suggested that higher contrasts -required for object detection in infrared imagery- are most likely to be observed at the boundaries of materials from different $\alpha / \varepsilon$ group for this kind of LEO.

In Figure 2, dash lines showed the effect of 0.1 value change of $\alpha$ and $\varepsilon$ either individually or both resulting approximately in $10^{\circ} \mathrm{C}$ variation in the material surface temperature. As it can be seen, such small variations at the values of $\alpha$ and $\varepsilon$ (which would encapsulate the range for the most of the space thermal coating materials) did not induce significant effect as much as the $\alpha / \varepsilon$ ratio. This also shows the accuracy of our study which aims to give an idea for the space debris temperature profile without knowing its surface coatings in detail. In this context, the provided results are recommended to be used only as guidance since our study aimed to put the boundaries of Infrared Based Active Debris Removal (IR-ADR) for any space debris rather than a specific target. Therefore, target specific analysis by using our method is highly recommended for better accuracy in real applications. This analysis also showed that the target in such orbit will require a detector dynamic range approximately between $-40^{\circ} \mathrm{C}$ and $30^{\circ} \mathrm{C}$.

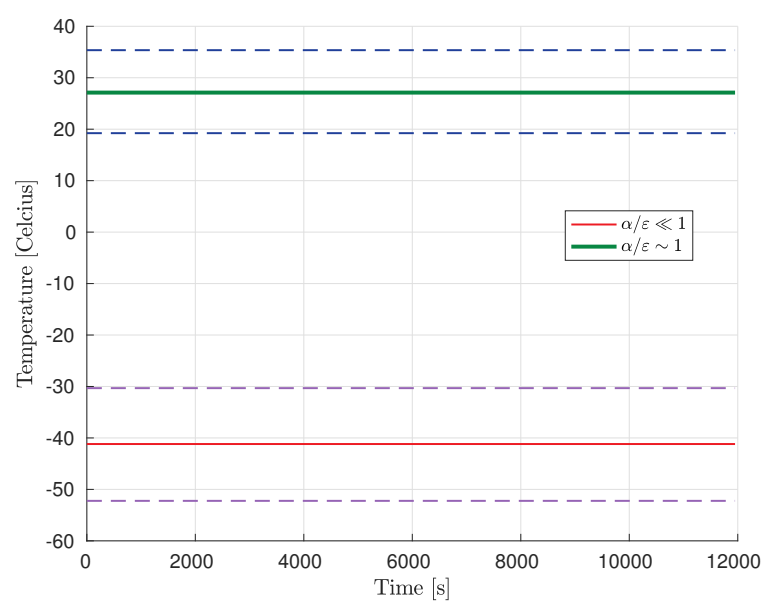

Fig. 2 Thermal variations of materials with $\alpha / \varepsilon \sim 1$ and $\alpha / \varepsilon \ll 1$ for Orbit Type - I. Dashed lines indicate the effect of $\alpha$ and/or $\varepsilon$ values change of 0.1 on temperature values

\subsection{Orbit Type-II}

In LEO, orbiting bodies can expose to eclipse up to one-third of their orbit such as SSO with Local Time of Ascending Node (LTAN) 10:30 at specific orbital configurations. Since these objects do cross the umbra cone, visual tracking of such targets gets interrupted by eclipse conditions. In this case, infrared based systems could provide the continuity of the operations and become more advantageous over their visual counterparts. However, their response to thermal environment variation had to be estimated in order to cover the target's thermal range and analyse its own challenges. In this context, the hottest and coldest temperatures needed to be computed. As the worst case scenario, we picked up SSO LTAN 10:30 with 30 minutes of eclipse and 100 minutes of total orbit duration as our representative orbit and approximated the albedo flux density as $45 \mathrm{~W} / \mathrm{m}^{2}$ from the mean values of ESATAN-TMS computations for one orbit. In Figure 3, first 2000s represented the entire eclipse which started again in 6000s.

As expected, the analysis showed that the highest temperatures were observed under solar illumination whereas being at their lowest at the end of eclipse period. The temperature variations of surface coatings along the orbit seem to be more drastic for materials with very low thermal inertia as it is shown in Figure 3. Moreover, the mean temperature of materials with $\alpha / \varepsilon \ll 1$ is found to be also lower comparing to materials with $\alpha / \varepsilon \sim 1$ as the absorbed radiation had been less than the emitted infrared radiation. This suggested that targets with homogeneous distribution of these two group of materials would provide better infrared features for target detection, recognition and tracking processes in ADR as they would provide the most distinct features.

Like in Orbit Type-I, the effect of small variations were also evaluated for Orbit Type-II. Similarly, the variation in temperature remained within $10^{\circ} \mathrm{C}$ throughout the orbit with slight increase in Sun illuminated regions of orbit. However, the margins represented with dashed lines in Figure 2 were not plotted in Figure 3 for clarity reasons.

The most significant outcome of this analysis was observed at periodic transitions from/to eclipse throughout the orbit. Although reaching the thermal steady state condition was longer for materials with thermally high inertia, the depicted contrast variations were found to be relatively faster -like down to a couple of minutes- due to low thermal inertia materials. As shown in Figure 3 with dashed ellipses, different types of material were observed at similar temperatures for a short while which was expected to cause the ambiguity in infrared detection since the thermal coatings generally have similar emissivity values. In other words depending on the thermal resolution of the infrared detector, some parts of the space debris might not be distinguishable for a while during the eclipse transition regardless of the detection algorithm performances as the number of debris image features would decrease. In the next section, this ambiguity problem will again be addressed in more detail. 


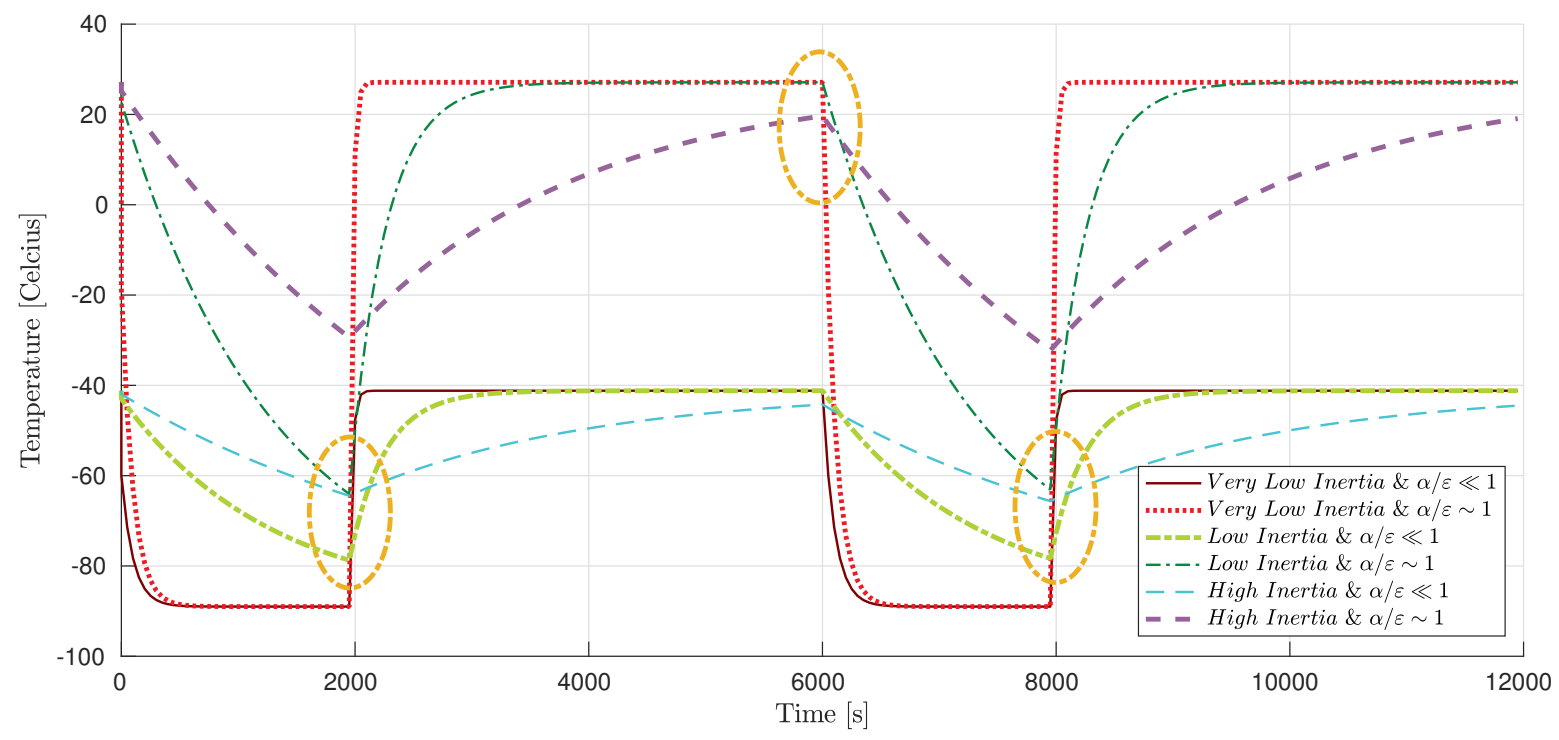

Fig. 3 Thermal variation of object with different surface coatings orbiting in Orbit Type-II throughout two orbits.

Beside this ambiguity caused by temporal variations noticed in Figure 3, an infrared scene with different surface coatings at a given time seems to have quite good contrast most of the time which would provide more texture for target recognition. Considering the thermal fluctuations along two representative orbit, the analysis also showed that the target in such orbit would require infrared detector with dynamic range approximately between $-90^{\circ} \mathrm{C}$ and $30^{\circ} \mathrm{C}$.

\subsection{Orbit Type-III}

In order to understand the effect of eclipse duration in thermal profile, the analysis was also performed for an orbit with half eclipse duration of Orbit Type-II i.e. SSO LTAN 08:00 with 16 minutes of eclipse and 100 minute of total orbit duration. Similar to Orbit Type-II, first 1000s represented the entire eclipse which started again in 6000s. The albedo flux of this orbit was approximated as $25 \mathrm{~W} / \mathrm{m}^{2}$ from the mean values of ESATAN-TMS computations for one orbit. As it was in Orbit Type-III, the main heat flux which caused the dynamic thermal environment for Orbit Type-II was Sun.

The analysis showed a similar trend as Orbit TypeII (Figure 3). The temperature of the materials with low inertia changed faster to steady state temperatures similarly whereas high inertia materials slowly as it can be seen in Figure 4. For an infrared imager, this effect could be visualized as in Figure 5. As it can be seen, the contrast during the transition is not static (variations in the sequence of frames) and in the case of tracking features arising from such contrast would not be stable. Such phenomena of ambiguity was considered to be important for IR-ADR and therefore discussed in next section in more details.

\section{Infrared Band Selection \& Infrared Signatures}

In the previous section, the dynamic range of temperature which space debris could encounter was estimated as $30^{\circ} \mathrm{C}$ to $-40^{\circ} \mathrm{C}, 30^{\circ} \mathrm{C}$ to $-90^{\circ} \mathrm{C}$ and $30^{\circ} \mathrm{C}$ to $-90^{\circ} \mathrm{C}$ for Orbit Type-I, Orbit Type-II and Orbit Type-III respectively. The colder the material got, the less radiation is emitted and it is harder to detect by infrared imagers due to noise. Therefore selecting the right thermal band is important and application specific.

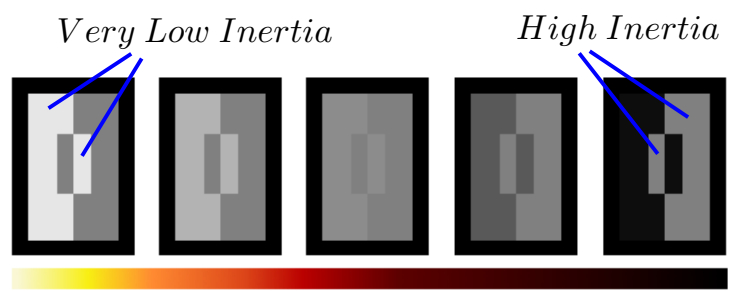

Fig. 5 Simulating contrast change at the boundaries of materials with High and Low Thermal Inertia during Eclipse transitions. Left to right is the transition from full illumination to eclipse conditions.

Since Orbit Type-II covered the entire temperature range of all three cases, the IR band selection for ADR navigation applications was based on this scenario. Figure 6 shows the blackbody radiation of object at different temperatures $\left(-90^{\circ} \mathrm{C}, 30^{\circ} \mathrm{C}\right.$ and $\left.5780^{\circ} \mathrm{K}\right)$ along the electromagnetic spectrum. The area below each temperature curve gives the amount of total radiated flux at given temperatures. The spectral bands of infrared for SWIR, MWIR and LWIR are defined as $1.5 \mu m-4 \mu m, 4 \mu m-8 \mu m$ and $8 \mu m-14 \mu m$. For instance, the total amount of radiation from an object at $-90^{\circ} \mathrm{C}$ in LWIR band is given by the shaded are in Figure 6. 


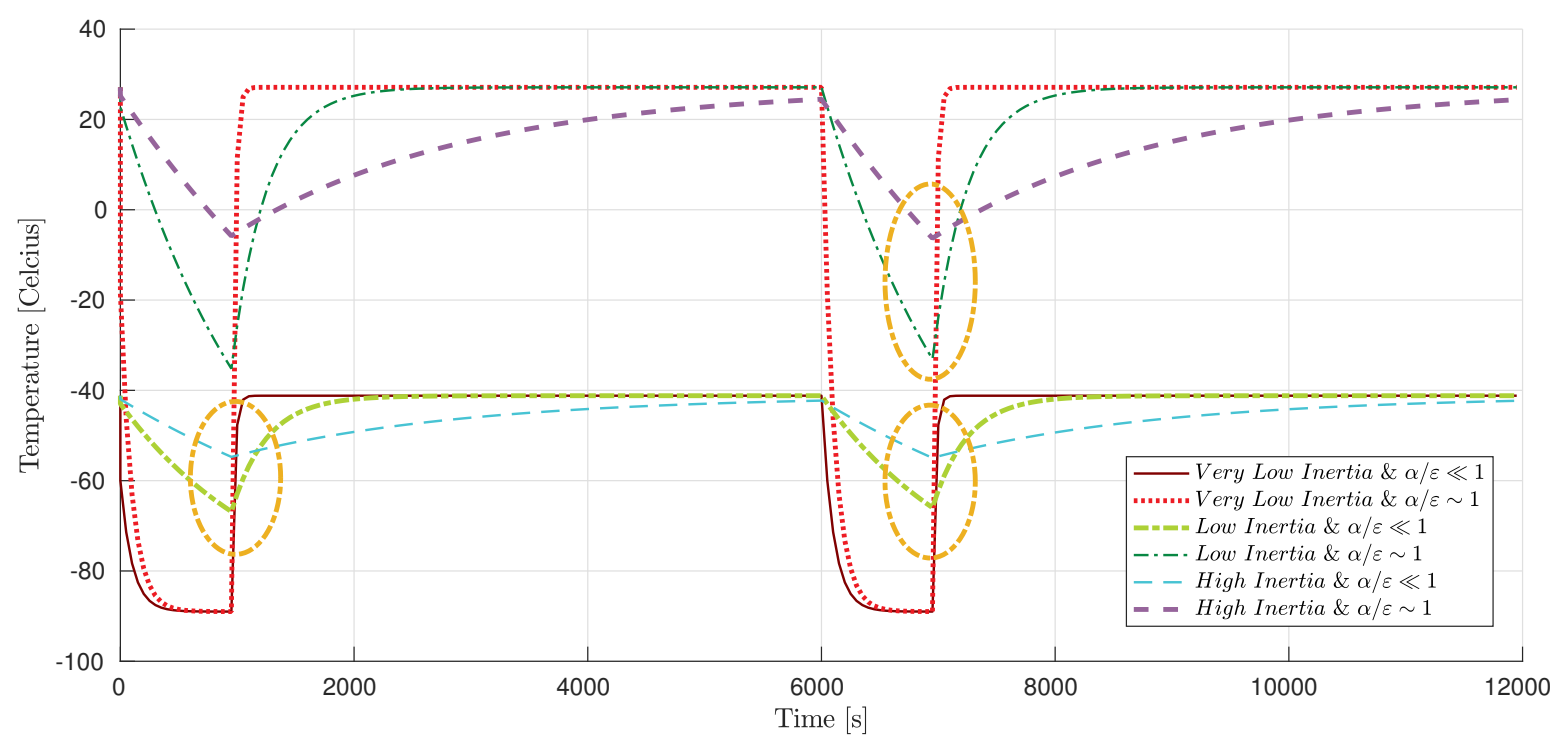

Fig. 4 Thermal variation of object with different surface coatings orbiting in Orbit Type-III throughout two orbits

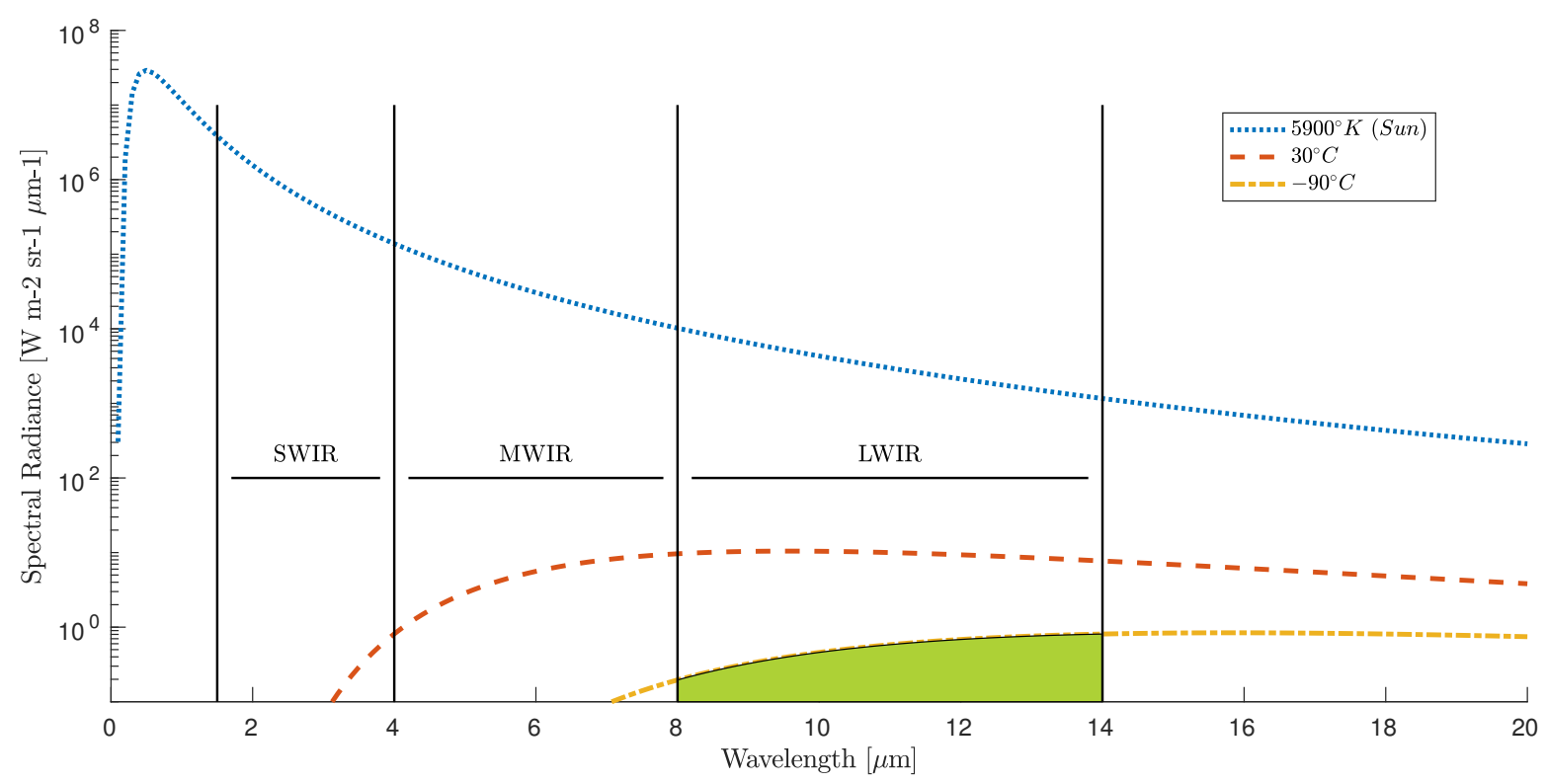

Fig. 6 Blackbody radiations for different temperatures. Green shaded area represents the total amount of LWIR band radiation from an object at $-90^{\circ} \mathrm{C}$

As the temperature decreases, the emitted flux decreases respectfully. For an object temperature below $-90^{\circ} \mathrm{C}$, there were no significant radiation in SWIR or MWIR comparing to LWIR. The highest flux for the given temperature range was observed for LWIR band.

For a detailed evaluation, the corresponding wavelength of highest radiation for given temperature range of $-90^{\circ} \mathrm{C}$ $30^{\circ} \mathrm{C}$ was computed by the Wien's displacement law

$$
\lambda_{\text {peak }}=\frac{2.898 \times 10^{3} \mu m K}{T} \quad[\mu m]
$$

where $T$ is the object blackbody temperature. As it was shown in Figure 7, blackbody with temperatures between $-90^{\circ} \mathrm{C}$ and $30^{\circ} \mathrm{C}$ which were the expected temperatures of space debris orbiting in Orbit Type-I and Orbit TypeII, radiates the most in LWIR region $(\lambda: 8 \mu m-14 \mu m)$. Therefore, LWIR was expected to be the best candidate that could provide good Signal to Noise Ratio (SNR) performance for ADR application.

With higher SNR levels, it is easier to distinguish objects from the electronic noise or the background scene (e.g. Earth at $27^{\circ} \mathrm{C}$ ) therefore better autonomous tracking capability can be achieved with minimum computational power. For ADR relative navigation applications, the coldest temperatures were considered as the design constraints since the main goal was to distinguish debris throughout the entire orbit regardless of illumination conditions since the infrared signatures of materials and the background which is deep space background $\left(2.7^{\circ} K\right)$ are very low. The practical limit 


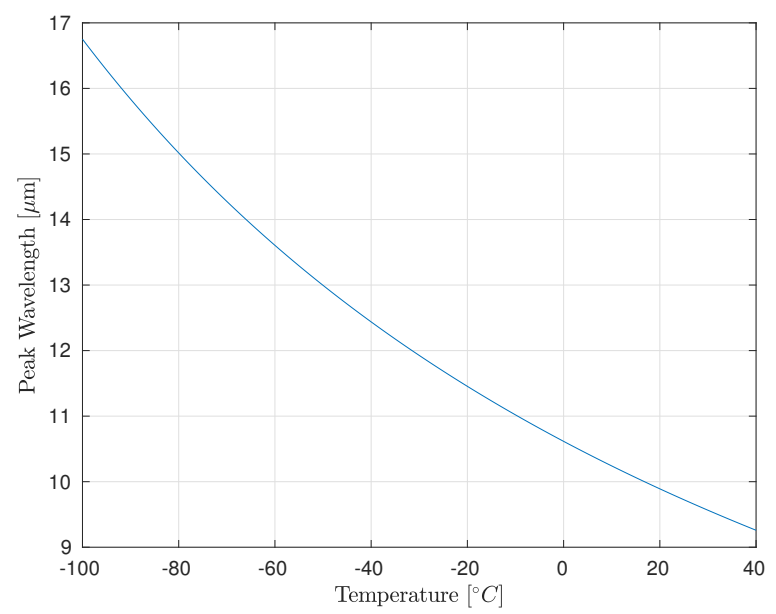

Fig. 7 Wavelength $\left(\lambda_{\text {peak }}\right)$ for peak radiation of given temperature

of minimum resolvable temperature by infrared detectors was related to detector's electronic noise. This means that the electronic noise would set the minimum level of resolvable contrast such as one between the debris in eclipse and deep space background. In order to have better performance in colder temperatures, the goal would be to increase the SNR as much as possible which would increase the contrast and therefore relax the constraints on image processing algorithms. With all this in mind, LWIR had been recommended for IR-ADR applications.

Figure 8 gives the amount of received radiation for three different bands within LWIR: $(8 \mu m-10 \mu m)$, $(10 \mu m-12 \mu m)$ and $(12 \mu m-14 \mu m)$ as well as the percentage of $(12 \mu m-14 \mu m)$ over total emission of $(8 \mu m-14 \mu m)$ band. Besides the fact that the amount of received power decreases with the temperature, most of the observed power was from higher wavelengths. In the temperature range of our interest, such small improvement in bandwidth towards higher wavelengths i.e. $(12 \mu m-14 \mu \mathrm{m})$ increases the level of signal radiated by the target up to $46 \%$. This found to be especially important for our test case of target temperatures reaching very low temperatures where the electronic noise would be significant.

It is also important to note that the percentage of $12 \mu \mathrm{m}-$ $14 \mu \mathrm{m}$ band contribution varies with the object blackbody temperature as it can be seen in Figure 8.

In summary, among all LWIR (i.e. $(\lambda: 8 \mu m-14 \mu m))$ band provided the higher signal level for Space Debris in Orbit Type-I, Orbit Type-II and Orbit Type-III where the temperature of space debris varied between $-90^{\circ} \mathrm{C}$ to $30^{\circ} \mathrm{C}$.

\subsection{Signature Ambiguities}

In infrared imaging, different materials could have similar signatures due to unfortunate combinations of temperatures

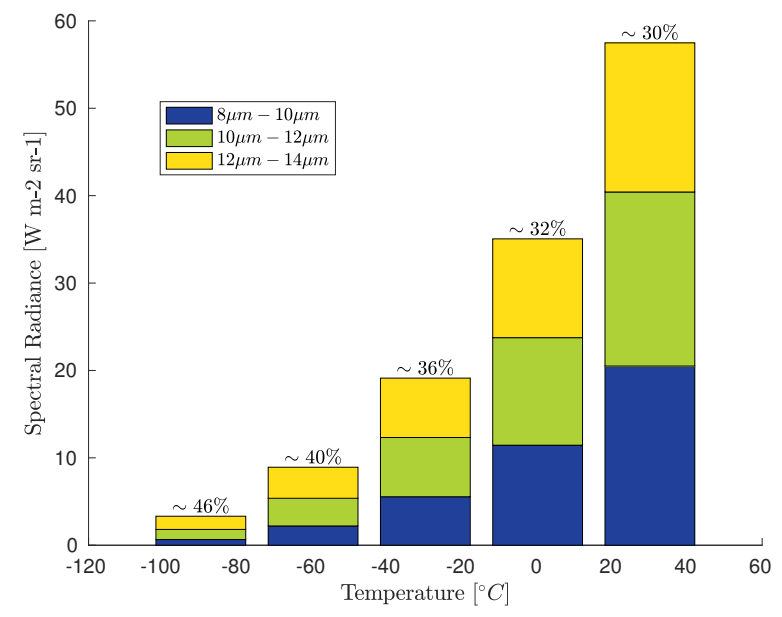

Fig. 8 Contribution of $12 \mu m-14 \mu m$ band to radiated power for different blackbody temperatures as percentage of $8 \mu m-14 \mu m$ range. Percentages are given on the top of each temperature range.

and emissivity which would cause ambiguities for detection algorithms. Previous analysis pointed out that such case would most likely to happen during the eclipse transitions as depicted with dashed ellipses in Figure 3 or small temperature fluctuations due to slight changes in $\alpha / \varepsilon$ as depicted with dashes boundaries in Figure 2. It is important to note that computed thermal profiles within the study would reflect the general overview of the space debris. However, the uncertainties in the surface coating material properties may also result in similar infrared signatures. In those cases where the emitted flux would be similar (e.g. A material with slightly lower temperature with reasonably higher emissivity compared to its surroundings where the infrared signatures would be the same.), the existing contrast between those materials would be ambiguous regardless of detector's sensitivity as depicted in the Figure 5.

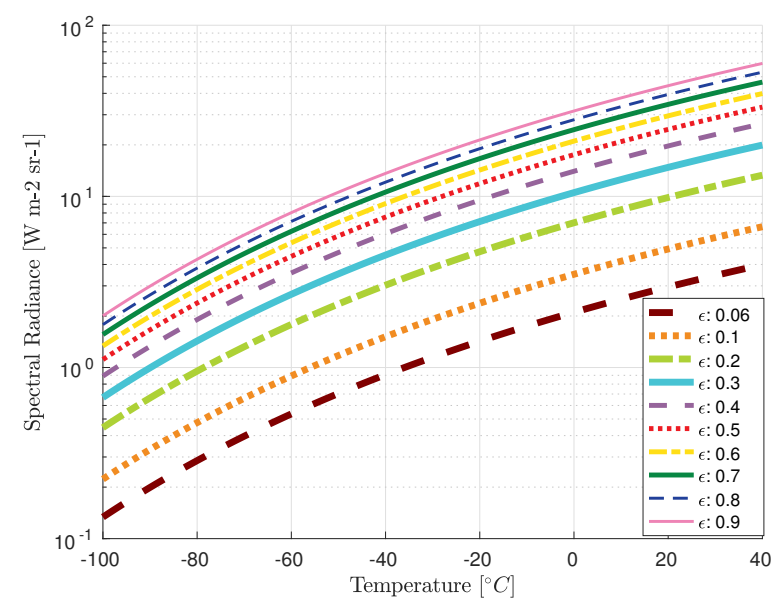

Fig. 9 Radiance Flux ambiguity of materials with different emissivity values at different temperatures

In order to evaluate the effects of such phenomena, the radiation of materials in LWIR (i.e. $(8 \mu m-14 \mu m)$ ) with 
different temperatures and emissivity had been computed as in Figure 9. This shows that bigger thermal differences would be more effective on distinguishing the infrared signatures at lower temperatures. At higher temperatures, thermal signatures of materials with closer $\varepsilon$ values would require bigger temperature differences to have a decent contrast compared to cases when their temperatures were lower. For instance the boundary between two materials with the $\varepsilon$ values 0.8 and 0.7 respectively would only be ambiguous when the temperature difference is $5^{\circ} \mathrm{C}$ and they are around $-90^{\circ} \mathrm{C}$. However, the boundary between same materials would be ambiguous with the difference of $10^{\circ} \mathrm{C}$ when they are around $10^{\circ} \mathrm{C}$. This suggested that boundaries of similar materials (similar emissivities) or materials with similar temperature profiles would not be good features to track in the case of space debris detection as they would not be stable. The case of boundary between different materials with different temperature profile could be visualised as Figure 5. In Figure 5, the space debris considered to be under Sun illumination (first frame) and heading to umbra (last frame). As found out earlier, the temperature profile of "very low thermal inertia" materials changed faster than materials with "high thermal inertia". Regardless of emissivity values of materials, this difference in thermal profile would cause the ambiguity -shown as the third frame in Figure 5 at some point of transition. Here it is important to note that the intensity value representing the "high thermal inertia" material remained the same throughout the illustration time for clarity reasons. However, such assumption of thermal stability for a given duration would not be too bad if one considers their thermal profile throughout one orbit.

\subsection{Infrared Signature of a Sample Space Debris}

The variety and the distribution of surface coating for man made space debris are unique to their design and our study aimed to provide a generic guideline on how to estimate their signature for given orbital parameters and material types. However, the performed analysis was considered to be more useful for the general interest. In this context, a hypothetical space debris model so called "EnvYsat", based on the mechanical thermal model of a former remote sensing satellite ENVISAT ${ }^{34}$ has been constructed for our experiments. The EnvYsat has similar type of surface coating with ENVISAT but with simpler geometry while providing a good representation of space debris. Based on our thermal analysis and a possible ADR trajectory, the CAD model of EnvYsat was amended with surface temperature of extreme cases i.e. just before eclipse and the end of eclipse state, from our estimated thermal profile. Then this model is placed into a possible orbital scenario created in the Synthetic Infrared Image Generator ${ }^{35}$ and the infrared scenes were simulated for a commercial off the shelf LWIR camera.

In Figure 10, the top scenes simulated the space debris with the deep space background $\left(2.7^{\circ} \mathrm{K}\right)$ and the below scenes with the constant Earth background $\left(27^{\circ} \mathrm{C}\right)$. The left scenes represent the space debris during a favourable targetchaser geometry configuration respect to Sun where all materials had reached their hottest steady state conditions in Orbit Type-II and would be referred as "Hot Case" hereafter. The coldest configuration (i.e. just before leaving the umbra cone) had been represented with the right scenes and would be referred as "Cold Case" hereafter.

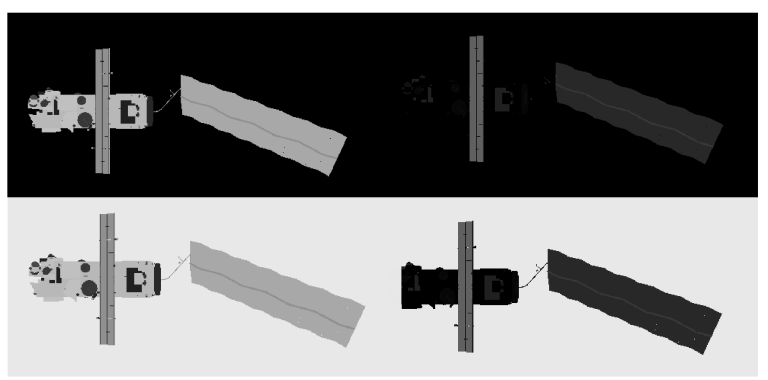

Fig. 10 Possible Scenes with different background and eclipse conditions

The results showed that the debris would have the least amount of texture -therefore the most unfavourable condition- in eclipse with the deep space background as given in the top right frame in Figure 10. As it can be seen, the outline of the space debris would be almost invisible to capture due to low signal levels suggesting the algorithm shall rely on parts of space debris with high thermal inertia. The highest amount of texture had been expected to be observed in the Hot Case with deep space background. In the case of Earth background which would happen during the fly-around phase of ADR, the extraction of the space debris silhouette was suggested to be challenging due to similar temperatures of Earth background and "low inertia" materials. However, the Earth had been expected to be more textured in LWIR which might allow contrast in some areas. Among all, the eclipse condition was found to be the most challenging due to the low level of signal which should be considered carefully for the continuity of the operation.

For the completeness of our study, we also would like to point out another challenge of infrared modality in space debris tracking. Unlike visual cameras, in ADR applications detected power by infrared systems mainly depends on the radiated power rather than the reflected light. This means 


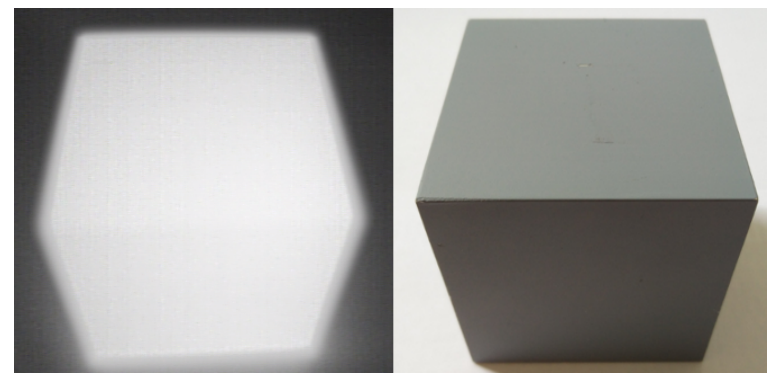

Fig. 11 Painted metal cube seen by LWIR camera(left), visible(right)

that infrared modality may lack an important information source coming from Sun-target-observer geometry so called shadow in ADR due to surface coatings' material properties as the contribution of their emitted thermal inertia might be significant comparing to reflected infrared radiation. This fact can be observed by the images captured by different modalities in Figure 11. In both images, the metal cube had been at same temperature in non-vacuum environment at our Unmanned Autonomous Systems Laboratory using our set-up of LWIR detector and Visual camera with properties given at Table 2. Since the polished aluminium would reflect the heat flux coming from our LWIR camera, we painted a metal cube with same colour in all faces in order to eliminate this effect in our imagery which would be negligible for ADR applications. Here, it is important to note that the halo around the cube depicted by the Figure 11(left) was due to thermal convection which would not be present in Space environment therefore shall not be expected to be observed. From Figure 11, it can be concluded that a simple edge based model tracking algorithm could possibly fail or diverge from the solution in IR-ADR mission for a tumbling target. This is due to the fact that as long as there is no temperature difference within the vicinity of non-coplanar surface, the infared signature from different point of view would be the same. In other words, tumbling motion of particular geometries could have singularities in their infrared signature at certain view points. However, this issue can be taken care of by using a robust tracking estimator or a complementary sensor suite like a LIDAR for a robust relative navigation solution.

Table 2. Camera Properties

\begin{tabular}{lll}
\hline Model & mvBlueFOX-ICG & FLIR Tau2 \\
\hline Resolution & $640 \times 480 \mathrm{pix}$ & $640 \times 512 \mathrm{pix}$ \\
Spectral band & $0.3-0.8 \mu \mathrm{m}$ & $7.5-13.5 \mu \mathrm{m}$ \\
\hline
\end{tabular}

\section{Conclusions and Future Work}

In this work, we presented an innovative grouping system for thermal surface coatings based on their behaviour in Space environment. By using this grouping, we presented a space debris infrared signature estimation method and verified this method with our infrared simulators and laboratory experiments for different orbital scenarios.

For the Orbit Type-I where there was no eclipse, the temperature profile was found to be stable and the values showed dependency on $\alpha / \varepsilon$ ratios. On the other hand, the Orbit Type-II and Orbit Type-III with various eclipse duration performed a very dynamic thermal profile throughout their orbit where the thermal inertia of the materials played an important role. Moreover, materials with different thermal inertia had been also found out to create challenging contrast variations throughout the orbital period. To the best of our knowledge, such complete thermal analysis has not been performed and infrared signature estimation methods of space debris to be used in ADR relative navigation solution have not been proposed before.

The performed research also showed that the LEO targets of IR-ADR Missions would have surface temperatures at the range of $-90^{\circ} \mathrm{C}$ and $+30^{\circ} \mathrm{C}$ in which the LWIR detectors would perform the best. Moreover, the importance of wider waveband had been underlined.

The challenges of infrared imaging in ADR applications had been presented as the result of our analysis. It has been shown that the detection algorithms used in infrared based relative navigation for ADR need to be robust and be able to deal with infrared signature ambiguities caused by surface coating material behaviours in space and target relative thermal signature respect to the different backgrounds. Given the number of parameters and uncertainties, finding the exact infrared signature of a space debris seems hardly feasible, but our study showed a simplified yet sufficient simulation for relative navigation solution design.

As a future work, the findings of this study will be used to create a representative simulation environment to develop robust tracking algorithms to be used in infrared based relative navigation solutions for ADR applications. Next, the possibility of a relative navigation solution without using an exact infrared appearance model of space debris will be investigated.

\section{Funding}

This work was supported by European Space Agency and SODERN under Network Partnership Initiative program [grant number NPI 342-2014]. 


\section{Declaration of Conflicting Interests}

The authors declare that there is no conflict of interest.

\section{Acknowledgements}

The authors would like to thank Guillermo Ortega and Mark Richardson for their valuable discussions.

\section{References}

1. Kessler DJ, Johnson NL, Liou J et al. The Kessler Syndrome : Implications to Future Space operations. Advances in the Astronautical Sciences 2010; 137: 47-61.

2. Liou JC. An active debris removal parametric study for LEO environment remediation. Advances in Space Research 2011; 47(11): 1865-1876. DOI:10.1016/j.asr.2011. 02.003. URL http://www.sciencedirect.com/ science/article/pii/s0273117711000974.

3. Klinkrad H. Space Debris. Encycloped ed. Wiley Online Library, 2010.

4. Krisko PH. The predicted growth of the low-Earth orbit space debris environment an assessment of future risk for spacecraft. Proceedings of the Institution of Mechanical Engineers, Part G: Journal of Aerospace Engineering 2007; 221(6): 975-985 DOI:10.1243/09544100JAERO192. arXiv:1011.1669v3.

5. Yaglioglu B, Yilmaz Ö, Utku A et al. Surveillance of Space: an Overview and a Vision for Turkey's Roadmap. 6th International Conference on Recent Advances in Space Technologies (RAST) 2013; : 1041-1046.

6. Yilmaz Ö and Imre E. Space-Based Surveillance of GEO Using GALILEO Constellation. 63rd International Astronautical Congress 2012; : 1-6.

7. Liou JC, Johnson $\mathrm{N}$ and Hill N. Controlling the growth of future LEO debris populations with active debris removal. Acta Astronautica 2010; 66(5-6): 648-653. DOI:10.1016/j.actaastro.2009.08.005. URL http://www.sciencedirect.com/science/ article/pii/s0094576509003981.

8. Wormnes K, Letty RL, Summerer L et al. ESA Technologies For Space Debris Remediation. Proceedings of the 6th IAASS Conference: Safety is Not an Option 2006; .

9. Fehse W. Rendezvous With and Capture / Removal of Noncooperative Bodies in Orbit The Technical Challenges. Journal of Space Safety Engineering 2014; 1(1): 17 - $27 . \quad$ DOI: 10.1016/S2468-8967(16)30068-4.

10. Maediger B, Sommer J, Ahrns I et al. RTES: Robotic Technologies for Space Debris Removal. International Symposium on Artificial Intelligence, Robotics and Automation in Space i-SAIRAS 2014; : 1-8.
11. Kervendal E, Chabot $\mathrm{T}$ and Kanani K. GNC Challenges and Navigation Solutions for Active Debris Removal Mission. Advances in Aerospace Guidance, Navigation and Control: Selected Papers of the Second CEAS Specialist Conference on Guidance, Navigation and Control 2013; : 761-779.

12. Kucharski D, Kirchner G, Koidl F et al. Attitude and spin period of space debris envisat measured by satellite laser ranging. IEEE Transactions on Geoscience and Remote Sensing 2014; 52(12): 7651-7657. DOI:10.1109/TGRS.2014. 2316138. TGRS.2014.2316138.

13. Fehse W. Automated Rendezvous and Docking of Spacecarft. 1 ed. Cambridge: Cambridge University Press, 2003.

14. Brooker G. Bolometers. In Introduction to Sensors for Ranging and Imaging. SciTech Publishing, 2009. pp. 82-83.

15. Sato T. Shape estimation of space debris using single-range doppler interferometry. IEEE Transactions on Geoscience and Remote Sensing 1999; 37(2 II): 1000-1014. DOI:10.1109/36. 752218.

16. Dennehy CJ. Relative Navigation Light Detection and Ranging (LIDAR) Sensor Development Test Objective (DTO) Performance Verification. Technical Report May, NASA Langley Research Center, 2013.

17. Kolb FM, Windmüller M, Rößler M et al. The LIRIS-2 3D Imaging LIDAR On ATV-5. 13th Symposium on Advanced Space Technologies - ASTRA 2015; (1): 3-6.

18. Polites ME. Technology of Automated Rendezvous and Capture in Space. Journal of Spacecraft and Rockets 1999; 36(2): 280-291.

19. Matko D, Rodič T, Blazič S et al. Lessons Learned From SPACE-SI Experiments on PRISMA Misson. 45th International Conference on Spacecraft Formation Flying Missions \& Technologies 2013; .

20. Mulder TA. ORBITAL EXPRESS Autonoumous Rendezvous And Capture Flight Operations Part 2 of 2 : AR\&C Exercises 4, 5 , and End-Of-Life. AIAA/AAS Astrodynamics Specialist Conference and Exhibit 2008; (August): 1-22. DOI:10.2514/ 6.2008-6768.

21. Deloo JAF and Mooij E. Active debris removal : Aspects of trajectories , communication and illumination during final approach. Acta Astronautica 2015; 117: 277-295. DOI: 10.1016/j.actaastro.2015.08.001. URL http://dx.doi. org/10.1016/j.actaastro.2015.08.001.

22. Yamamoto T, Murakami N, Nakajima $Y$ et al. Navigation and Trajectory Design For Japanese Active Debris Removal Mission. International Symposium on Space Flight Dynamics 2014; (May).

23. Strube M, Henry R, Skelton E et al. RAVEN: An on-orbit relative navigation demonstration using international space station visiting vehicles. American Astronautical Society 2015; 
24. Luu T, Ruel S and Labrie M. TriDAR Test Results Onboard Final Shuttle Mission, Applications for Future of NonCooperative Autonomous Rendezvous \& Docking. 11th International Symposium on Artificial Intelligence, Robotics, and Automation in Space (i-SAIRAS) 2012; .

25. Cavrois B, Vergnol A, Donnard A et al. LIRIS demonstrator on ATV5 : a step beyond for European non cooperative navigation system. AIAA Guidance, Navigation, and Control Conference 2015; .

26. Shi Jf, Ulrich S, Ruel $S$ et al. Uncooperative Spacecraft Pose Estimation Using an Infrared Camera During Proximity Operations. AIAA SPACE 2015 Conference and Exposition 2015; : 1-17.

27. McCall P, Sharples R, Andrian JH et al. Thermal modeling of space debris via Finite Element Analysis. Proceeding of the Advanced Maui Optical and Space Surveillance Technologies Conference 2013; (1).

28. McCall PD, Naudeau ML and Adjouadi M. Debris characterization techniques via unresolved longwave infrared imaging from a space-based platform. Journal of Applied Remote Sensing 2014; 8(1): 084989. DOI:10.1117/1.JRS.8.084989. URL http:

//remotesensing.spiedigitallibrary.org/ article.aspx?doi=10.1117/1.JRS.8.084989.

29. Vallado DA. Fundamentals of Astrodynamics and Applications. 3rd ed. New York: Microcosm Press, 2004.

30. Wie B. Earth Satellite Applications. In Space Vehicle Dynamics and Control. 2008. pp. 253-254.

31. Gilmore DG. Spacecraft Thermal Control Handbook Volume 1 Fundamental Technologies. 2 ed. American Institute of Aeronautics and Astronautics/Aerospace Press., 2002.

32. Tsai JR. Overview of Satellite Thermal Analytical Model. Journal of Spacecraft and Rockets 2004; 41(1).

33. ITP Engines Ltd UK. ESATAN-TMS Thermal Modelling Suite. [Online]. Available from: https://www. esatan-tms.com/. [Accessed 26-06-2016].

34. Louet $\mathrm{J}$ and Bruzzi S. ENVISAT mission and system. Proceedings of Geoscience and Remote Sensing Symposium 1999; .

35. Richard Ayling, Brian Butters, Nic Millwood RW. Simultaneous IR and RF modelling and simulation of platforms, threats and countermeasures using CounterSim. AOC International Exhibition and Symposium 2008; . 